\title{
Saddle Embolism in the Pulmonary Artery Bifurcation: Massive Pulmonary Embolism
}

Md Atiquzzaman ${ }^{*}$, Mohammad Omar Faruq², Kazi Nuruddin Ahmed³, Md Ashikuzzaman Sohan ${ }^{4}$, Rifat Zaman $^{4}$, Farzana Akhtar ${ }^{5}$

A 60-year-old smoker, hypertensive and diabetic male presented to the emergency department of a multidisciplinary hospital in Dhaka with complaints of severe shortness of breath for 2 hours and chest pain for 3 hours. Initial clinical examination revealed respiratory rate- $28 / \mathrm{min}$, pulse- $120 / \mathrm{min}$, blood pressure- $80 / 50 \mathrm{~mm}$ of $\mathrm{Hg}, \mathrm{SpO}_{2}-88 \%$ with $\mathrm{O}_{2} 10 \mathrm{~L} / \mathrm{min}$ and bilateral scattered wheeze in both lung field. No other significant finding was noted, and he was immediately shifted to intensive care unit (ICU) for further management. Immediate chest X-ray was normal, hematology \& biochemistry were non-remarkable, ECG-sinus tachycardia with right ventricular strain pattern. Cardiac bio-markers were also normal, there was metabolic acidosis and respiratory alkalosis with moderate hypoxemia in arterial blood gas (ABG) analysis, D-dimer was elevated (positive in qualitative measurement). Based on these, with a presumed diagnosis of pulmonary embolism, computed tomographic pulmonary angiography (CTPA) was done which reveled almost total occlusion at pulmonary artery bifurcation (Image $1 \& 2$ ). Color Doppler echocardiogram showed dilated right ventricle and moderate pulmonary arterial hypertension. Immediate anticoagulation with unfractionated heparin followed by streptokinase thrombolysis owing to hemodynamic instability (requiring inotrope support). He also required mechanical ventilation. The patient expired on the following day.

1. Senior Clinical Staff, General ICU, Asgar Ali Hospital, Dhaka-1204, Bangladesh.

2. Professor \& Chief Consultant, General ICU and Emergency, United Hospital Ltd., Dhaka-121, Bangladesh.

3. Specialist, General ICU, Asgar Ali Hospital, Dhaka-1204, Bangladesh.

4. Senior Resident Medical Officer, General ICU, Asgar Ali Hospital, Dhaka-1204, Bangladesh.

5. Senior Medical Officer, Department of Obst. \& Gynae, OGSB Hospital \& Institute of Reproductive \& Child Health, Dhaka, Bangladesh.

*Corresponding Author:

Dr. Md. Atiquzzaman

MBBS

Senior Clinical Staff, General ICU, Asgar Ali Hospital

111/1/A, Distillery Road, Gandaria, Dhaka-1204, Bangladesh.

E-mail: drazrinku@gmail.com

Contact: +8801711939036

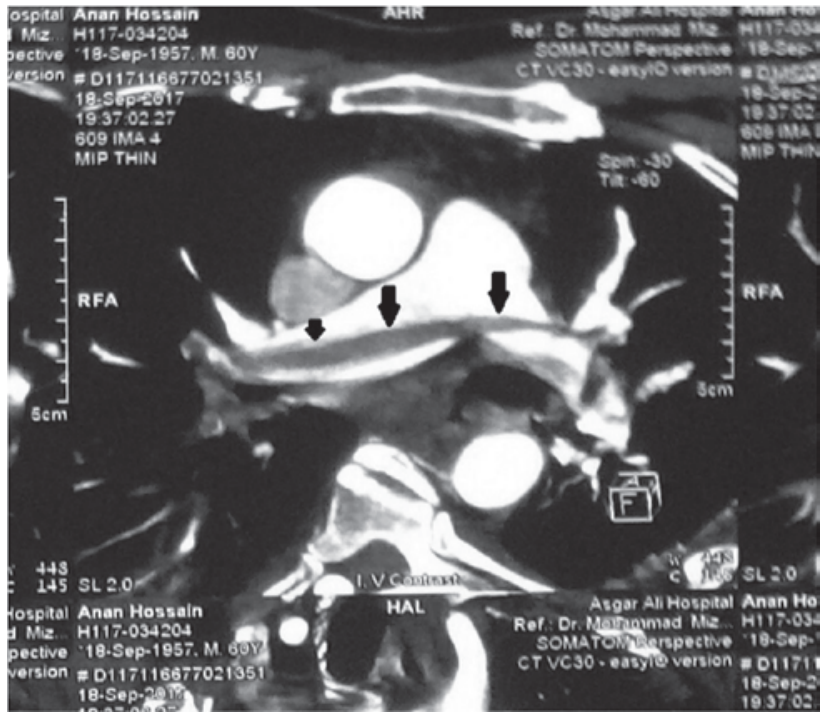

Image 1: Arrows in 2D axial CTPA images point to a saddle embolism in the pulmonary artery bifurcation.

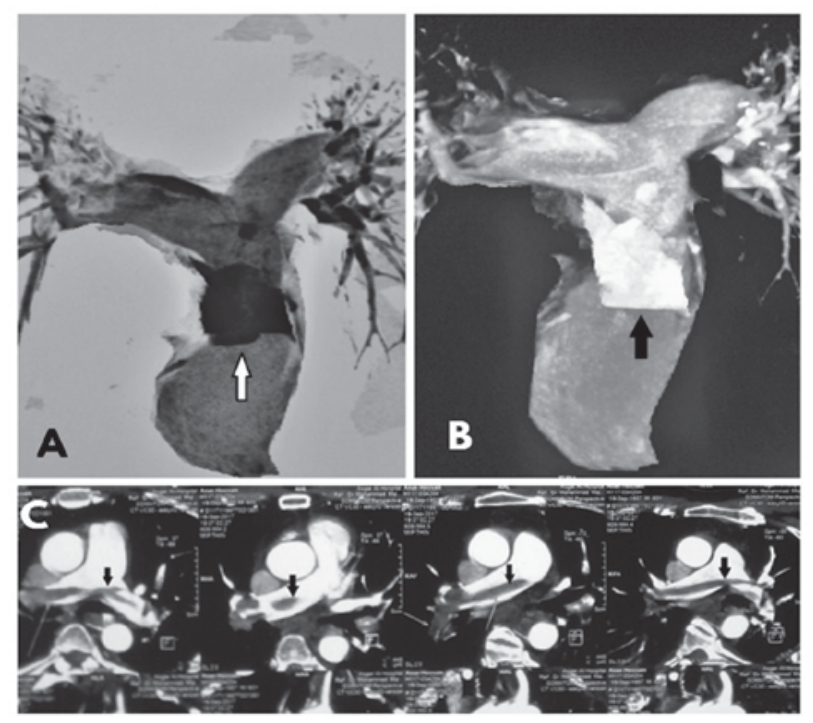

Image 2 A, B \& C: Arrows in 2D CTPA images showing filling defect (large thrombus) in the pulmonary artery.

\section{DISCUSSION}

Pulmonary embolism (PE) is a common and potentially fatal disease, especially among the elderly but is still underdiagnosed $d^{1,2,6}$. Incidence rate increases exponentially 
with age for both men and women. The overall age-adjusted incidence rate is higher for men (114 per 100000) than women (105 per 100000), male: female sex ratio is 1.2:1. Incidence rate is somewhat higher in women during the childbearing years. For almost one-quarter of PE patients, the initial clinical presentation is sudden death ${ }^{1}$. The majority of PE arise from the propagation of lower limb deep vein thrombosis. Rare causes include septic emboli (from endocarditis affecting the tricuspid or pulmonary valves), tumor (especially choriocarcinoma), fat emboli following fracture of long bones, air, and amniotic fluid - which may enter mother's circulation following delivery ${ }^{3,7}$. Risk factors convincingly demonstrated include male gender, increasing patient age and body mass index, major surgery, prolonged immobility, hospital or nursing home confinement, active cancer with or without concurrent chemotherapy, multiple trauma, chronic heart failure, central vein catheterization or transvenous pacemaker, prior thrombosis, varicose veins, neurological disease with leg paresis and few hematological conditions; patients with chronic liver disease have a reduced risk $^{1,5}$. A recognized risk factor is present in $80-90 \%{ }^{3}$. Clinical presentation is non-specific, depends on number, size and distribution of emboli and on underlying cardiorespiratory reserve $^{3,7}$. Most patients with PE feature at least one of four symptoms which, in decreasing order of frequency, are sudden onset dyspnea, chest pain, fainting (or syncope), and hemoptysis. The occurrence of such symptoms, if not explained otherwise, should alert the clinicians to consider PE in differential diagnosis, and order the appropriate objective test ${ }^{6}$. The patients with suspected pulmonary embolism must undergo some diagnostic tests until the diagnosis is ascertained or eliminated or an alternative diagnosis is confirmed. The simplified Well's pre-test probability scoring algorithm for pre-investigation evaluation of $\mathrm{PE}$ is a commonly utilized and validated assessment tool ${ }^{4}$. An elevated D-dimer is of limited value, as it may be raised in a variety of other conditions, including myocardial infarction, pneumonia and sepsis. However, low levels, particularly in the context of a low clinical risk, have a high negative predictive value $(99 \%)$ and limit further investigation or anticoagulation ${ }^{3,8}$. A variety of non-specific chest radiographic abnormalities have been described associated with acute PE including normal, cardiomegaly, pleural effusion, elevated hemidiaphragm, pulmonary artery enlargement, atelectasis, and parenchymal pulmonary infiltrates; although cardiomegaly is the most common finding 9 . Arterial blood gases typically show a reduced $\mathrm{PaO} 2$ and a normal or low $P a \mathrm{CO} 2$, may be normal in a significant minority. A metabolic acidosis often seen in acute massive PE with cardiovascular collapse $^{3}$. ECG is useful in excluding other differential diagnoses, such as acute myocardial infarction or pericarditis. The most common findings in PE include sinus tachycardia and $\mathrm{T}$ wave inversion in the precordial leads which are non-specific; massive emboli cause right heart strain revealed by an $\mathrm{S}_{1} \mathrm{Q}_{3} \mathrm{~T}_{3}$ pattern, ST-T changes and appearance of right bundle branch block ${ }^{3,10}$. Transthoracic color Doppler echocardiography rarely confirms a diagnosis of pulmonary embolism by visualizing a thrombus. However, easily observed morphological and Doppler abnormalities may result from acute pulmonary hypertension: dilatation of the right heart chambers, a very sensitive and reliable sign of severe pulmonary embolism when the ratio of the right/left ventricular dimension $>0.6$, abnormal interventricular septal contraction, a very specific sign of massive pulmonary embolism, and increased systolic pulmonary artery pressure. Echocardiography is extremely helpful in differential diagnosis and assessment of circulatory collapse ${ }^{3,11}$. CTPA is the gold standard diagnostic test ${ }^{6,7}$. Nephrotoxicity and history of allergy to contrast media limits it's use. In these cases, ventilation-perfusion (V/Q) scanning or ventilation-perfusion single photon emission computed tomography (V/Q SPECT) may be considered. Duplex ultrasound or Venogram of leg veins may demonstrate the source ${ }^{3,7}$. Prompt recognition and treatment potentially life-saving. Adequate oxygenation, maintenance of circulation with intravenous fluids or plasma expander and alleviation of symptoms with opiates is immediate focus. Main principle of treatment is anticoagulation. Inferior vena caval filters may be used in patients in whom anticoagulation is contraindicated or recurrent thromboembolism despite anticoagulation. Thrombolysis or surgical pulmonary embolectomy is indicated in patients with acute massive PE accompanied by cardiogenic shock ${ }^{3}$. The risk of recurrence is high. So, persons at risk for venous thromboembolism must first be identified ${ }^{1,3}$.

$\mathrm{PE}$ remains a disease which requires high clinical suspicion. Unlike other conditions, no specific symptoms, signs or investigations reflect a disease process immediately. Hence patients presenting with acute onset of dyspnea should be evaluated for common cause of dyspnea but pulmonary embolism should be kept in mind, so as not to miss this fatal disease.

\section{References:}

1. Heit JA. The epidemiology of venous thromboembolism in the community. Arteroscler Thromb Vasc Biol. 2008; 28: 370-2. DOI: 10.1161/ATVBAHA.108.162545.

2. Silverstein MD, Heit JA, Mohr DN, Petterson TM, O'Fallon WM, Melton LJ 3rd. Trends in the incidence of deep vein thrombosis and pulmonary embolism: a 25-year population-based study. Arch Intern Med. March 1998; 158(6): 585-93. DOI: 10.1001/archinte.158.6.585.

3. Reid PT, Innes JA. Respiratory Medicine. In: Stuart H. Ralston, Ian D. Penman, Mark WJ Strachan, and Richard P Hobson. Davidson's principles \& practice of medicine. $23^{\text {rd }}$ ed. Edinburg: Churchill Livingstone Elsevier; 2014. p. 619-20.

4. Gibson NS, Sohne M, Kruip MJ, Tick LW, Gerdes VE, Bossuyt PM et al ; Christopher study investigators. Further validation and simplification of the Wells clinical decision rule in pulmonary embolism. Thromb Haemost 2008; 99(01): 229-234. DOI: 10.1160/TH07-05-0321.

5. Anderson FA Jr, Spencer FA. Risk factors for venous thromboembolism. Circulation 2003; 107:I9-16. DOI: 10.1161/01.CIR.0000078469.07362.E6. 
6. Miniati M, Cenci C, Monti S, Poli D. Clinical Presentation of Acute Pulmonary Embolism: Survey of 800 Cases. PLoS One. February 2012; 7(2): e30891. DOI: 10.1371/journal.pone.0030891.

7. Wells PS, Ginsberg JS, Anderson DR, Kearon C, Gent M, Turpie AG et al. Use of clinical model for safe management of patients with suspected pulmonary embolism. Ann Intern Med 1998; 129: 997-1005. DOI: 10.7326/0003-4819-129-12-199812150-00002.

8. Sendama W, Musgrave KM. Decision-Making with D-Dimer in the Diagnosis of Pulmonary Embolism. The American Journal of Medicine. December 2018; 131(12): 1438-1443. DOI: https://doi.org/10.1016/j.amjmed.2018.08.006.
9. Elliot CG, Goldhaber SZ, Visani L, De Rosa M. Chest radiography in acute pulmonary embolism. Results from the International Cooperative Pulmonary Embolism Registry. Chest 2000; 118: 33-8. DOI: $10.1378 /$ chest.118.1.33

10. Ferrari E, Imbert A, Chevalier T, Mihoubi A, Morand P, Baudouy M. The ECG in pulmonary embolism. Predictive value of negative T waves in precordial leads -80 case reports. Chest 1997; 111:537-43. DOI: 10.1378/chest.111.3.537.

11. Augusseau-Richard MP, Pacouret G, Dessenne X, Charbonnier B. Echocardiography in pulmonary embolism. Arch Mal Coeur Vaiss. Nov 1995; 88(11 Suppl): 1715-22. 